\title{
The Application of Zhang-Gradient Method for Iterative Learning Control
}

\author{
Zhang Qunli \\ Department of Mathematical and Statistical, Heze University, Heze, Shandong 274015, P. R. China \\ Email: qunli-zhang@126.com
}

\begin{abstract}
The novel sufficient conditions for nonlinear systems without and with time-delay, whose initial state are zero or not, are studied using the $\lambda$-norm, Zhang-gradient method and retarded Gronwall-like inequality. An examples is shown the effectiveness of the mentioned technique.
\end{abstract}

Keywords: Iterative learning control, Zhang-gradient method, tracking error; convergence, time delay

\section{Introduction}

Iterative learning control methodology, which is proposed by Arimoto et al. in 1984 (See[1]), is to utilize the previous control information of the studied systems. The repetitive behavior has been a major research area and a hot issue in recent years (See[2-15]).

Recently, Zhang-gradient method has shown their powerful performance in solving online timevarying control problem (See[16-20]). The Zhang-gradient method is based on an indefinite errormonitoring function (See[20]), but the gradient dynamics method is usually designed from a norm or square-based energy function.

Stabilization problem of control systems has received some research results and have been reported in the literature[3,11,13,15,21-29]. However, only a few results combining with the iterative learning control items and Zhang-gradient method are available for nonlinear systems. In this paper, under the case that the initial state $y_{k}(0)=y_{k+1}(0)$ or $y_{k}(0) \neq y_{k+1}(0)$, the iterative learning controller of nonlinear systems is designed by using Zhang-gradient method and $\lambda$-norm.

\section{Preliminaries}

Throughout this paper, $\|x\|=\left(\sum_{i=1}^{n} x_{i}^{2}\right)^{1 / 2}$ is said to be the 2-norm for the vector $x=\left(x_{1}, x_{2}, \ldots, x_{n}\right)^{T}$, while the $\lambda$-norm for the $x(t)$ function is defined as $\|x(t)\|_{\lambda}=\sup _{t \in[0, T]}\left\{e^{-\lambda t}\|x(t)\|\right\}$, where $\lambda>0$.

Lemma. [10,30] Consider $\sup _{t \in[0, T]}\left\{e^{-\lambda t} \int_{0}^{t}\left\|_{x}(t)\right\|_{d \tau}\right\} \leq \frac{1}{\lambda}\left\|_{x}(t)\right\|_{\lambda}$.

\section{Design of Iterative Learning Controller of Nonlinear Systems}

In this section, we will take account of two cases about the iterative initial state vector $y_{k+1}(0)=y_{k}(0)$ and $y_{k+1}(0) \neq y_{k}(0)$, respectively, of nonlinear systems without and with time delay.

Theorem 1. Consider iterative learning control on the system

$$
\dot{y}_{k}(t)=f\left(t, y_{k}(t)\right)+u_{k}(t)
$$

where $y_{k}(t) \in R^{n}$ is the $k$ th iterative state vector, $f\left(t, y_{k}(t)\right)$ is the nonlinear operator $[0, T] \times R^{n} \rightarrow R^{n}$, and satisfies

$$
\left\|f\left(t, y_{k+1}(t)\right)-f\left(t, y_{k}(t)\right)\right\| \leq l_{f}\left\|y_{k+1}(t)-y_{k}(t)\right\|
$$


$u_{k}(t)$ is the $k$-th iterative control input, $T$ is a constant.

When $y_{k+1}(0)=y_{k}(0)$, the iterative learning controller is designed as

$$
u_{k+1}(t)=u_{k}(t)+m \hbar\left(e_{k}(t)\right)
$$

When $y_{k+1}(0) \neq y_{k}(0)$, the iterative learning controller is designed as

$$
u_{k+1}(t)=u_{k}(t)+m \hbar\left(e_{k}(t)\right)+\psi_{k, h}(t)\left(y_{k+1}(0)-y_{k}(0)\right)
$$

where $e_{k}(t)=y_{d}(t)-y_{k}(t)$ is Zhang function (i.e., error function), $y_{d}(t)$ is a desired output, $\hbar\left(e_{k}(t)\right)$ is a monotonically increasing odd function, $m<0$ is a constant, and $\int_{0}^{t} \psi_{k, h}(s) d s=1$.

Besides, according to Zhang-gradient method (See $[31,32]$ ), the formula can be used

$$
\dot{e}_{k}(t)=-\mu \hbar\left(e_{k}(t)\right), \quad \mu>0
$$

If there exist a constant $\lambda>0$ such that

$$
\left(\frac{|\mu+m|}{\mu}+\frac{l_{f}}{\mu} \cdot \frac{|m|}{\lambda-l_{f}}\right)<1
$$

then the system (2) can guarantee that $\left\|y_{d}(t)-y_{k}(t)\right\|$ is bounded and $y_{k}(t)$ can track $y_{d}(t)$ on $t \in[0, T]$, i.e. $\lim _{k \rightarrow+\infty} y_{k}(t)=y_{d}(t)$.

Proof. From $e_{k}(t)=y_{d}(t)-y_{k}(t)$, we know that $e_{k+1}(t)=e_{k}(t)+y_{k}(t)-y_{k+1}(t)$. So

$$
\dot{e}_{k+1}(t)=\dot{e}_{k}(t)+\dot{y}_{k}(t)-\dot{y}_{k+1}(t)
$$

According to (2), (3) and (6), we have

$$
\begin{aligned}
&-\mu \hbar\left(e_{k+1}(t)\right)=-\mu \hbar\left(e_{k}(t)\right)+f\left(t, y_{k}(t)\right)+u_{k}(t)-f\left(t, y_{k+1}(t)\right)-u_{k+1}(t) \\
&=-\mu \hbar\left(e_{k}(t)\right)-m \hbar\left(e_{k}(t)\right)+\left[f\left(t, y_{k}(t)\right)-f\left(t, y_{k+1}(t)\right)\right] \\
&=-(\mu+m) \hbar\left(e_{k}(t)\right)+\left[f\left(t, y_{k}(t)\right)-f\left(t, y_{k+1}(t)\right)\right] \\
& \hbar\left(e_{k+1}(t)\right)=\frac{(\mu+m)}{\mu} \hbar\left(e_{k}(t)\right)+\frac{1}{\mu}\left[f\left(t, y_{k+1}(t)\right)-f\left(t, y_{k}(t)\right)\right] \\
&\left\|\hbar\left(e_{k+1}(t)\right)\right\| \leq \frac{|\mu+m|}{\mu}\left\|\hbar\left(e_{k}(t)\right)\right\|+\frac{l_{f}}{\mu}\left\|y_{k+1}(t)-y_{k}(t)\right\|
\end{aligned}
$$

Taking $\lambda$-norm, we have

$$
\left\|\hbar\left(e_{k+1}(t)\right)\right\|_{\lambda} \leq \frac{|\mu+m|}{\mu}\left\|\hbar\left(e_{k}(t)\right)\right\|_{\lambda}+\frac{l_{f}}{\mu}\left\|y_{k+1}(t)-y_{k}(t)\right\|_{\lambda}
$$

From iterative law (4), (5) and Lemma,

$$
\begin{aligned}
& y_{k+1}(t)-y_{k}(t)=\int_{0}^{t}\left(\dot{y}_{k+1}(\tau)-\dot{y}_{k}(\tau)\right) d \tau+\left(y_{k}(0)-y_{k+1}(0)\right) \\
&=\int_{0}^{t}\left(f\left(t, y_{k+1}(t)\right)-f\left(t, y_{k}(t)\right)\right) d t+\int_{0}^{t}\left(u_{k+1}(t)-u_{k}(t)\right) d t+\left(y_{k}(0)-y_{k+1}(0)\right) \\
&=\int_{0}^{t}\left(f\left(t, y_{k+1}(\tau)\right)-f\left(t, y_{k}(\tau)\right)\right) d \tau+\int_{0}^{t} m \hbar\left(e_{k}(\tau)\right) d \tau \\
&\left\|y_{k+1}(t)-y_{k}(t)\right\| \leq \int_{0}^{t} l_{f}\left\|y_{k+1}(\tau)-y_{k}(\tau)\right\| d \tau+\int_{0}^{t}|m|\left\|\hbar\left(e_{k}(\tau)\right)\right\| d \tau \\
&\left\|y_{k+1}(t)-y_{k}(t)\right\|_{\lambda} \leq \frac{l_{f}}{\lambda}\left\|y_{k+1}(t)-y_{k}(t)\right\|_{\lambda}+\frac{|m|}{\lambda}\left\|\hbar\left(e_{k}(t)\right)\right\|_{\lambda} \\
&\left\|y_{k+1}(t)-y_{k}(t)\right\|_{\lambda} \leq \frac{|m|}{\lambda-l_{f}}\left\|\hbar\left(e_{k}(t)\right)\right\|_{\lambda}
\end{aligned}
$$

where $\lambda>l_{f}$. 
From (8) and (9),

$$
\begin{aligned}
\left\|\hbar\left(e_{k+1}(t)\right)\right\|_{\lambda} & \leq \frac{|\mu+m|}{\mu}\left\|\hbar\left(e_{k}(t)\right)\right\|_{\lambda}+\frac{l_{f}}{\mu} \cdot \frac{|m|}{\lambda-l_{f}}\left\|\hbar\left(e_{k}(t)\right)\right\|_{\lambda} \\
& =\left(\frac{|\mu+m|}{\mu}+\frac{l_{f}}{\mu} \cdot \frac{|m|}{\lambda-l_{f}}\right)\left\|\hbar\left(e_{k}(t)\right)\right\|_{\lambda}
\end{aligned}
$$

When the condition (7) is true, $\lim _{k \rightarrow+\infty}\left\|\hbar\left(e_{k}(t)\right)\right\|_{\lambda}=0$. That $\hbar\left(e_{k}(t)\right)$ is a monotonically increasing odd function implies $\lim _{k \rightarrow+\infty}\left\|e_{k}(t)\right\|_{\lambda}=0$, i.e. $\lim _{k \rightarrow+\infty}\left\|e_{k}(t)\right\|=0$.

Theorem 2. Consider the following system

$$
\dot{y}_{k}(t)=f\left(t, y_{k}(t)\right)+g\left(t, y_{k}(t-\tau)\right)+u_{k}(t)
$$

where $y_{k}(t) \in R^{n}$ is the $k$-th iterative state vector, $f\left(t, y_{k}(t)\right), g\left(t, y_{k}(t-\tau)\right)$ are the operator $[0, T] \times R^{n} \rightarrow R^{\mathrm{n}}$, and satisfy

$$
\begin{gathered}
\left\|f\left(t, y_{k+1}(t)\right)-f\left(t, y_{k}(t)\right)\right\| \leq l_{f}\left\|y_{k+1}(t)-y_{k}(t)\right\| \\
\left\|g\left(t, y_{k+1}(t-\tau)\right)-g\left(t, y_{k}(t-\tau)\right)\right\| \leq l_{g}|| y_{k+1}(t-\tau)-y_{k}(t-\tau) \|
\end{gathered}
$$

$u_{k}(t)$ is the $k$-th iterative control input, $\tau>0$ is time delay, and $T$ is a constant. When $y_{k+1}(0)=y_{k}(0)$, the iterative learning controller is designed as (4). When $y_{k+1}(0) \neq y_{k}(0)$, the iterative learning controller is designed as (5). The Zhang gradient design formula can be used as (6). The other conditions are the same with Theorem 1 . If there exist a constant $\lambda>0$ and a continuous function $\varphi(t) \neq 0, t \in[0, T]$, such that

$$
\left(\frac{|\mu+m|}{\mu}+\frac{l_{f}}{\mu} \cdot \frac{e^{\left(l_{f}+l_{g}\right) t}|m|}{\lambda}+\frac{l_{g}}{\mu} \cdot \frac{e^{\left(l_{f}+l_{g}\right)(t-\tau)}|m|}{\lambda} \cdot \frac{\varphi(t-\tau)}{\varphi(t)}\right)<1
$$

then the system (10) can guarantee that $\left\|y_{d}(t)-y_{k}(t)\right\|$ is bounded and $y_{k}(t)$ can track $y_{d}(t)$ on $t \in[0, T]$, i.e. $\lim _{k \rightarrow+\infty} y_{k}(t)=y_{d}(t)$.

Proof. From $e_{k}(t)=y_{d}(t)-y_{k}(t)$, we know that $e_{k+1}(t)=e_{k}(t)+y_{k}(t)-y_{k+1}(t)$, so $\dot{e}_{k+1}(t)=\dot{e}_{k}(t)+\dot{y}_{k}(t)-\dot{y}_{k+1}(t)$, that is

$$
\begin{aligned}
-\mu \hbar\left(e_{k+1}(t)\right)= & -\mu \hbar\left(e_{k}(t)\right)+f\left(t, y_{k}(t)\right)+g\left(t, y_{k}(t-\tau)\right)+u_{k}(t) \\
& -f\left(t, y_{k+1}(t)\right)-g\left(t, y_{k+1}(t-\tau)\right)-u_{k+1}(t) \\
= & -\mu \hbar\left(e_{k}(t)\right)-m \hbar\left(e_{k}(t)\right)+\left[f\left(t, y_{k}(t)\right)-f\left(t, y_{k+1}(t)\right)\right] \\
& +\left[g\left(t, y_{k}(t-\tau)\right)-g\left(t, y_{k+1}(t-\tau)\right)\right] \\
= & -(\mu+m) \hbar\left(e_{k}(t)\right)+\left[f\left(t, y_{k}(t)\right)-f\left(t, y_{k+1}(t)\right)\right] \\
& +\left[g\left(t, y_{k}(t-\tau)\right)-g\left(t, y_{k+1}(t-\tau)\right)\right] \\
\hbar\left(e_{k+1}(t)\right)= & \frac{(\mu+m)}{\mu} \hbar\left(e_{k}(t)\right)+\frac{1}{\mu}\left[f\left(t, y_{k+1}(t)\right)-f\left(t, y_{k}(t)\right)\right] \\
& +\frac{1}{\mu}\left[g\left(t, y_{k+1}(t-\tau)\right)-g\left(t, y_{k}(t-\tau)\right)\right] \\
\| e_{k+1} & (t))\left\|\leq \frac{|\mu+m|}{\mu}\right\| \hbar\left(e_{k}(t)\right)\left\|+\frac{l_{f}}{\mu}\right\| y_{k+1}(t)-y_{k}(t) \| \\
& +\frac{l_{g}}{\mu}\left\|y_{k+1}(t-\tau)-y_{k}(t-\tau)\right\|
\end{aligned}
$$

Taking $\lambda$-norm, we have

$$
\left\|\hbar\left(e_{k+1}(t)\right)\right\|_{\lambda} \leq \frac{|\mu+m|}{\mu}\left\|\hbar\left(e_{k}(t)\right)\right\|_{\lambda}+\frac{l_{f}}{\mu}\left\|y_{k+1}(t)-y_{k}(t)\right\|_{\lambda}+\frac{l_{g}}{\mu}\left\|y_{k+1}(t-\tau)-y_{k}(t-\tau)\right\|_{\lambda}
$$




$$
\begin{aligned}
y_{k+1}(t)-y_{k}(t)= & \int_{0}^{t}\left(\dot{y}_{k+1}(\tau)-\dot{y}_{k}(\tau)\right) d \tau+\left(y_{k}(0)-y_{k+1}(0)\right) \\
= & \int_{0}^{t}\left(\dot{y}_{k+1}(\tau)-\dot{y}_{k}(\tau)\right) d \tau \\
= & \int_{0}^{t}\left(f\left(t, y_{k+1}(\tau)\right)-f\left(t, y_{k}(\tau)\right)\right) d \tau+\int_{0}^{t}\left(u_{k+1}(\tau)-u_{k}(\tau)\right) d \tau \\
& +\int_{0}^{t}\left(g\left(s, y_{k+1}(s-\tau)\right)-g\left(s, y_{k}(s-\tau)\right)\right) d s \\
= & \int_{0}^{t}\left(f\left(t, y_{k+1}(\tau)\right)-f\left(t, y_{k}(\tau)\right)\right) d \tau+\int_{0}^{t} m \hbar\left(e_{k}(\tau)\right) d \tau \\
& +\int_{0}^{t}\left(g\left(s, y_{k+1}(s-\tau)\right)-g\left(s, y_{k}(s-\tau)\right)\right) d s \\
\left\|y_{k+1}(t)-y_{k}(t)\right\| \leq \int_{0}^{t}\left\|f\left(s, y_{k+1}(s)\right)-f\left(s, y_{k}(s)\right)\right\| d s+\int_{0}^{t}|m|\left\|\hbar\left(e_{k}(s)\right)\right\| d s & \\
& +\int_{0}^{t}\left\|g\left(s, y_{k+1}(s-\tau)\right)-g\left(s, y_{k}(s-\tau)\right)\right\| d s \\
& \leq l_{f}^{t} \int_{0}^{t}\left\|y_{k+1}(s)-y_{k}(s)\right\| d s+\int_{0}^{t}|m|\left\|\hbar\left(e_{k}(s)\right)\right\| d s \\
& +l_{g}^{t} \int_{0}^{t}\left\|y_{k+1}(s-\tau)-y_{k}(s-\tau)\right\| d s
\end{aligned}
$$

Utilizing the retarded Gronwall-like inequality [15,33]and Lemma, we obtain

$$
\begin{gathered}
\left\|y_{k+1}(t)-y_{k}(t)\right\| \leq e^{\left(l_{f}+l_{g}\right) t} \int_{0}^{t}|m|\left\|\hbar\left(e_{k}(s)\right)\right\| d s \\
\left\|y_{k+1}(t)-y_{k}(t)\right\|_{\lambda} \leq \frac{e^{\left(l_{f}+l_{g}\right) t}|m|}{\lambda}\left\|\hbar\left(e_{k}(s)\right)\right\|_{\lambda} \\
\left\|y_{k+1}(t-\tau)-y_{k}(t-\tau)\right\|_{\lambda} \leq \frac{e^{\left(l_{f}+l_{g}\right)(t-\tau)}|m|}{\lambda}\left\|\hbar\left(e_{k}(t-\tau)\right)\right\|_{\lambda}
\end{gathered}
$$

From (15)-(17), we obtain

$$
\left\|\hbar\left(e_{k+1}(t)\right)\right\|_{\lambda} \leq\left(\frac{|\mu+m|}{\mu}+\frac{l_{f}}{\mu} \cdot \frac{e^{\left(l_{f}+l_{g}\right) t}|m|}{\lambda}\right)\left\|\hbar\left(e_{k}(t)\right)\right\|_{\lambda}+\left(\frac{l_{g}}{\mu} \cdot \frac{e^{\left(l_{f}+l_{g}\right)(t-\tau)}|m|}{\lambda}\right)\left\|\hbar\left(e_{k}(t-\tau)\right)\right\|_{\lambda}
$$

It is easy to know that the solution of the equation $x_{k+1}(t)=\alpha x_{k}(t)+\beta x_{k}(t-\tau)$ is

$$
x_{k}(t)=c \varphi(t)\left(\alpha+\beta \frac{\varphi(t-\tau)}{\varphi(t)}\right)^{k}
$$

where $\alpha, \beta$ are given constants, $c$ is an arbitrary constant, $\varphi(t)$ is an arbitrary function and satisfies that $\frac{\varphi(t-\tau)}{\varphi(t)}$ is a constant.

From (19) and the condition (14), $\lim _{k \rightarrow+\infty}\left\|\hbar\left(e_{k}(t)\right)\right\|_{\lambda}=0$. That $\hbar\left(e_{k}(t)\right)$ is a monotonically increasing odd function implies $\lim _{k \rightarrow+\infty}\left\|e_{k}(t)\right\|_{\lambda}=0$, i.e. $\lim _{k \rightarrow+\infty}\left\|e_{k}(t)\right\|=0$.

\section{Example}

For further illustration, we consider the following system 


$$
\begin{aligned}
& \dot{y}_{k}(t)=A y_{k}(t)-B f\left(y_{k}(t-\tau)\right)+u_{k}(t), \\
& u_{k+1}(t)=u_{k}(t)+m e_{k}(t)-\psi_{k, h}(t)\left(y_{k}(0)-y_{k+1}(0)\right)
\end{aligned}
$$

where

$$
\begin{gathered}
A=\left(\begin{array}{cc}
0.6 \cos t & 0.02 \\
0.1 & 0.8 \sin t
\end{array}\right), B=\left(\begin{array}{cc}
0.5 & 0 \\
0 & 0.5
\end{array}\right), y_{k}(t)=\left(\begin{array}{l}
y_{1, k}(t) \\
y_{2, k}(t)
\end{array}\right), \\
f\left(y_{k}(t-\tau)\right)=\left(\begin{array}{l}
\left|y_{1, k}(t-1)+1\right|-\left|y_{1, k}(t-1)-1\right| \\
\left|y_{2, k}(t-1)+1\right|-\left|y_{2, k}(t-1)-1\right|
\end{array}\right)
\end{gathered}
$$

Taking $\quad m=-1.5, \quad \mu=2, \quad l_{\digamma}=1.43, \quad l_{g}=2, \quad \varphi(t)=0.2 e^{1.2 t}, \quad \psi_{k, h}(t)=\left\{\begin{array}{cc}\pi \cos (\pi t), & t \in[0,1] \\ 0, & t \in(1,2]\end{array}\right.$. From the above example, it can be easily proved that the condition (13) of Theorem 2 is satisfied.

\section{Conclusion}

In this paper, considering the iterative learning control problem for nonlinear systems without and with time delays, and combining with Zhang-gradient method, the novel controllers, which can guarantee the robust convergence of the tracking error, are designed.

Acknowledgments. The author were supported financially by the National Natural Science Foundation of China (11371221), the Natural Science Foundation of Shandong Province of China (ZR2014AM032), and the Project of Shandong Province Higher Educational Science and Technology Program (J16LI15).

\section{Reference}

1. S.Arimoto, S.Kawamura, and F.Miyazaki. Bettering operation of robots by learning, Journal of Robotic Systems, 1(2),pp.123-140,1984.

2. H.-S. Lee and Z. Bien, A note on convergence property of iterative learning controller with respect to sup norm, Automatica,vol.33,no.8,pp.1591-1593,1997.

3. Y. Chen, Z. Gong, and C. Wen, Analysis of a high-order iterative learning control algorithm for uncertain nonlinear systems with state delays, Automatica,vol.34,no.3,pp.345-353, 1998.

4. Sheng Liu, Changkui Xu, and Lanyong Zhang. Robust Course Keeping Control of a Fully Submerged Hydrofoil Vessel without Velocity Measurement: An Iterative Learning Approach, Mathematical Problems in Engineering, Article ID 7979438, Volume 2017, 14 pages ,2017.

5. Dongqi Ma, Hui Lin. An Accelerating Iterative Learning Control Based on an Adjustable Learning Interval, Journal of Control Science and Engineering, Article ID 1731676, Volume 2017,6 pages, 2017.

6. R.H.Chi,Z.S.Hou,andJ.X.Xu. AdaptiveILCforaclass of discrete-time systems with iteration-varying trajectory and random initial condition, Automatica, vol.44,no.8,pp.2207-2213, 2008.

7. Leila Noueili, Wassila Chagra, and Moufida Ksouri. New Iterative Learning Control Algorithm Using Learning Gain Based on $\sigma$ Inversion for Nonsquare Multi-Input Multi-Output Systems, Modelling and Simulation in Engineering, Article ID 4195938, Volume 2018, 9 pages ,2018.

8. Lei Li. Lebesgue-p Norm Convergence Analysis of PDo-Type Iterative Learning Control for Fractional-Order Nonlinear Systems,Discrete Dynamics in Nature and Society, Article ID 5157267, Volume 2018,10 pages, 2018.

9. Xiongfeng Deng, Xiuxia Sun, and Shuguang Liu. Consensus Learning Control for Leader-Following Nonlinear Multiagent Systems with Control Delay, Wireless Communications and Mobile Computing, Article ID 2035683, Volume 2019,10 pages,2019.

10. Xiongfeng Deng, Xiuxia Sun, Shuguang Liu, and Boyang Zhang. Leader-Following Consensus for Second-Order Nonlinear Multiagent Systems with Input Saturation via Distributed Adaptive Neural Network Iterative Learning Control, Complexity, Research Article (13 pages), Article ID 9858504, Volume 201913 pages, 2019.

11. Xiaoli Li, Jian Liu, Linkun Wang, Kang Wang, and Yang Li. Welding Process Tracking Control Based on 
Multiple Model Iterative Learning Control, Mathematical Problems in Engineering, Article ID 6137352, Volume 2019, 9 pages, 2019.

12. J. Li and J. Li. Iterative learning control approach for a kind of heterogeneous multi-agent systems with distributed initial state learning, Applied Mathematics and Computation, vol.265,pp.1044-1057, 2015.

13. L. Yan and J. Wei. Fractional order nonlinear systems with delay in iterative learning control, Applied Mathematics and Computation,vol.257,pp.546-552,2015.

14. H. Cai, Y. Huang, J. Du, T. Tang, D. Zuo, and J. Li. Iterative learning control with extended state observer for telescope sys-tem, Mathematical Problems in Engineering, vol.2015,Article ID 701510, 8 pages, 2015.

15. Zhang Qunli. The effect of initial state error for nonlinear systems with delay via iterative learning control, Advances in Mathematical Physics, Volume 2016, Article ID 461950, 6pages,2016.

16. J.Cao, Y.Wan. Matrix measure strategies for stability and synchronization of inertial BAM neural network with time delay, Neural Netw.53,pp.165-172,2014.

17. S.Das, A.Acharya, I.Pan. Simulation studies on the design of optimum PID controller to suppress chaotic oscillations in a family of Lorenz-like multi-wing attractors, Math.Comput. Simul,100,pp.72-87,2014.

18. C.Yi, Y.Zhang, D.Guo. A new type of recurrent neural networks for real-time solution of Lyapunov equation with time-varying coefficient matrices, Math. Comput. Simul, 92,pp.40-52,2013.

19. Y.Zhang,M.Li, Y.Yin,L.Jin, X.Yu. Controller design of nonlinear system for fully trackable and paths by combining ZD and GD, in:Proc.25 ${ }^{\text {th }}$ Control and Decision Conference,pp.209-214,2013.

20. Y.Zhang, C.Yi, D.Guo, J.Zheng. "Comparison on Zhang neural dynamics and gradient-based neural dynamics for online solution of nonlinear time-varying equation", Neural Comput. Appl. 20,pp.1-7,2011.

21. S.S.Ge,F.Hong,andT.H.Lee, Robust adaptive control of nonlinear systems with unknown time delays, Automatica, vol.41,no.7,pp.1181-1190,2005.

22. C. C. Hua, G. Feng, and X. P. Guan. Robust controller design of a class of nonlinear time delay systems via backstepping method, Automatica,vol.44,no.2,pp.567-573,2008.

23. X. D. Ye. Adaptive stabilization of time-delay feedforward nonlinear systems, Automatica,vol.47,no.5,pp.950$955,2011$.

24. J. Na. Adaptive prescribed performance control of nonlinear systems with unknown dead zone, International Journal of Adaptive Control and Signal Processing, vol.27, no.5,pp.426-446, 2013.

25. Z.-Y. Sun and Y.-G. Liu. Adaptive control design for a class of uncertain high-order nonlinear systems with time delay, Asian Journal of Control,vol.17,no.2,pp.535-543,2015.

26. Qunli Zhang. Synchronization of multi-chaotic systems via ring impulsive control, Control theory \& Applications,vol.27,no.2, pp. 226-232, 2010.

27. Qunli Zhang. Synchronization of multi-chaotic systems with ring and chain intermittent connections, Applied Mechanics and Materials,vol.241-244,pp.1081-1087,2013.

28. Q.Zhang. A class of vector Lyapunov functions for stability analysis of nonlinear impulsive differential systems, Mathematical Problems in Engineering, vol. 2014, Article ID 649012, 9 pages, 2014.

29. Q. Zhang. Matrix measure with application in quantized synchronization analysis of complex networks with delayed time via the general intermittent control, Annals of Applied Mathematics,vol.31,no.1,pp.115-126,2015.

30. M.X.Sun and B.J.Huang. Iterative learning control, National Defence Industry Press, Beijing, China,1999.

31. Zhang Y., Ma W., Cai B. From Zhang neural network to Newton iteration for matrix inversion, IEEE Trans. Circuits Syst. I, Regul. Pap,56(7),pp1405-1415,2009.

32. Zhang Y., Yi C. Zhang neural network and neural-dynamic method, Nova Science Publishers, New York,2011.

33. R.P.Agarwal, S.deng, and W. Zhang. Generalization of a retarded Gronwall-like inequality and its applications, Applied Mathematics and Computation,165(3),pp.599-612,2005. 